\title{
BMJ Open Factors related to receipt of non-cancer- related transurethral prostatectomy: findings from a large prospective study of 106769 middle-aged and older Australian men
}

\author{
Grace Joshy, ${ }^{1}$ Kay Soga,${ }^{1}$ Rosemary J Korda, ${ }^{1}$ Manish I Patel, ${ }^{2,3}$ Emily Banks ${ }^{1,4}$
}

To cite: Joshy G, Soga K, Korda RJ, et al. Factors related to receipt of noncancer-related transurethral prostatectomy: findings from a large prospective study of 106769 middle-aged and older Australian men. BMJ Open 2017;7:e013737. doi:10.1136/bmjopen-2016013737

- Prepublication history and additional material is available. To view please visit the journal (http://dx.doi.org/ 10.1136/bmjopen-2016013737).

Received 8 August 2016 Revised 3 January 2017 Accepted 12 January 2017

CrossMark

\begin{abstract}
${ }^{1}$ National Centre for Epidemiology and Population Health, Research School of Population Health, Australian National University, Canberra, Australian Capital Territory, Australia

${ }^{2}$ Discipline of Surgery, University of Sydney, Sydney, New South Wales, Australia ${ }^{3}$ Department of Urology, Westmead Hospital, Sydney, New South Wales, Australia ${ }^{4}$ The Sax Institute, Sydney, New South Wales, Australia
\end{abstract}

Correspondence to Dr Grace Joshy; grace.joshy@anu.edu.au

\section{ABSTRACT}

Background: Transurethral prostatectomy (TURP) is a common surgical intervention for chronic lower urinary tract symptoms (LUTS). Little large-scale evidence exists on factors related to receipt of non-cancer-related TURP.

Methods: A prospective study of men aged $\geq 45$ years participating in the 45 and Up Study, a large Australian cohort study, without prior prostatectomy and/or bowel/ genital/urinary-tract cancer; questionnaire data were linked to hospitalisations and deaths. HRs for TURP were estimated in relation to multiple factors, adjusting for confounders.

Results: There were 3416 incident TURPs among 106769 men (median follow-up 5.8 years), with rates of $1.8,5.3,9.1$ and $11.4 / 1000$ person-years for ages $45-54,55-64,65-74$ and $\geq 75$ years, respectively. Age-adjusted rates of TURP varied markedly according to baseline LUTS from 2.2/1000 person-years with no/mild symptoms to $30.7 / 1000$ person-years with severe symptoms. Annual household income $\geq \$ 70000$ versus $<\$ 20000$, having private health insurance and living in major cities were associated with higher TURP rates; there were no significant differences according to baseline diabetes, stroke, high blood pressure or cardiovascular disease. Men reporting severe versus no physical functioning limitation, high versus low psychological distress or poor versus excellent self-rated health were $36-51 \%$ more likely to undergo procedures overall, but were $24-37 \%$ less likely to undergo procedures following additional adjustment for need (baseline LUTS).

Conclusions: TURP rates were most strongly related to baseline LUTS and age, consistent with appropriate health services targeting. Lower TURP rates in men experiencing socioeconomic disadvantage and with poor health/disability, after accounting for baseline LUTS, suggest inequity and factors such as frailty and risks related to surgery.

\section{INTRODUCTION}

Lower urinary tract symptoms (LUTS) are responsible for considerable morbidity,

\section{Strengths and limitations of this study}

- Despite lower urinary tract symptoms (LUTS) being the most frequent reason for hospitalisation among men, there is little large-scale evidence on patterns of receipt of surgical intervention (transurethral prostatectomy (TURP)) in Australia or other countries with universal healthcare.

- This large data linkage study was able to prospectively investigate factors related to receipt of non-cancer-related TURP, accounting for age, the level of need for surgery and other potential confounding factors. The score used to measure LUTS at baseline has been calibrated against the validated and widely used international prostate symptom score. A relatively high rate of LUTS procedures relative to need was observed among men in high-income households, living in major cities and with private health insurance; this suggests inequality.

- The ability to link to administrative records allowed virtually complete ascertainment of outcomes over time; people with previous cancer could be objectively identified; bowel/genital/ urinary-tract cancer during follow-up could be censored as a competing risk.

- Limitations of the study include non-availability of data on the management of symptoms in clinical practice, lack of information on contraindications to surgical intervention and the use of self-reported survey data.

distress, reductions in health-related quality of life and healthcare costs. ${ }^{1}$ Although there are many causes of LUTS in men, including detrusor dysfunction, overactive bladder syndrome, urinary tract infections, prostatitis and malignancy, the most common cause is benign prostate enlargement (BPE), which obstructs the bladder outlet. There are many more LUTS-related surgical interventions attributable to LUTS than to malignancy. 
In Australia, procedures on the prostate during 20042005 included 21110 transurethral prostatectomies (TURPs), the majority of which (14 109) were for a principal diagnosis of hyperplasia of the prostate. $^{2}$ Healthcare systems around the world face increasing costs in managing BPE; it is estimated that the UK spends over 180 million pounds for BPE treatments each year; ${ }^{3}$ the direct cost of medical services for BPE in the USA was around $\$ 1.1$ billion in $2000 .{ }^{4}$

Despite their frequency and cost, LUTS and their surgical treatments remain under-researched and poorly quantified. There are surprisingly few population-based data available on the relationship of LUTS to surgical intervention rates, ${ }^{5}{ }^{6}$ and limited data on the factors relating to such surgery, taking into account the level of need. In addition, previous studies have focused on incidence and risk factors ${ }^{7}$ for LUTS in general; there is little evidence on factors related to the non-cancer-related surgery for LUTS, which is the most common type of procedure. ${ }^{9}$

The objectives of this study were to quantify the incidence of non-cancer-related TURP in relation to severity of LUTS, age and other sociodemographic and health-related characteristics, and to examine factors related to receipt of the TURP after accounting for LUTS severity, among men in the general population in Australia.

\section{METHODS}

The Sax Institute's 45 and Up Study is a large-scale Australian cohort study of 267153 men and women aged 45 years and over, randomly sampled from the general population of New South Wales (NSW), Australia. Individuals joined the study by completing a postal questionnaire (distributed from 1 January 2006 to 31 December 2008) and giving informed consent for follow-up through repeated data collection and linkage of their data to population health databases. The conduct of the 45 and Up Study was approved by the University of New South Wales Human Research Ethics Committee. The study methods are described in detail elsewhere. ${ }^{10}$

Questionnaire data from study participants have been linked probabilistically to hospitalisations and deaths by the Centre for Health Record linkage (http://www. cherel.org.au/). The linked data contain details of hospital admissions in participants from 1 July 2000 to 30 June 2014, including the primary reason for admission (up to 51 clinical diagnoses), ${ }^{11}$ and up to 50 procedures codes; ${ }^{12}$ see details in the online supplementary Technical Appendix. Dates of death were ascertained from the date of recruitment up to 18 June 2014.

Data on sociodemographic characteristics, health behaviours and health status, including LUTS, were derived from self-reported data from the baseline questionnaire available at: https://www.saxinstitute.org. au/our-work/45-up-study/questionnaires/. To measure
LUTS, we used the modified International Prostate Symptom Score (m-IPSS) ${ }^{8}$ see details in the online supplementary Technical Appendix. The m-IPSS has previously been calibrated against the original IPSS, ${ }^{8}$ allowing comparability between the two scores. The m-IPSS was categorised as 0-2, 3-5, 6-8, 9-11 and 12-21 (from no/mild symptoms to severe symptoms).

Physical functioning limitation was assessed by the Medical Outcomes Study-Physical Functioning scale (MOS-PF) ${ }^{13}$ Psychological distress was measured using the Kessler Psychological Distress Scale (K10). ${ }^{14}$ Self-rated health was measured using the response categories of 'excellent', 'very good', 'good', 'fair' or 'poor'. Body mass index (BMI) was classified as underweight $\left(15-<18.5 \mathrm{~kg} / \mathrm{m}^{2}\right)$, healthy weight $\left(18.5-<25 \mathrm{~kg} / \mathrm{m}^{2}\right)$, overweight $\left(25-<30 \mathrm{~kg} / \mathrm{m}^{2}\right)$ and obese $\left(30-50 \mathrm{~kg} / \mathrm{m}^{2}\right)$. Assessment of baseline sociodemographic factors and health conditions is outlined in the online supplementary Technical Appendix.

\section{Statistical methods}

This study includes men who entered the 45 and Up Study between 2006 and 2009; there were 123820 men after excluding those with linkage errors $(\mathrm{n}=22)$ and those aged $<45$ years at baseline $(n=5)$. Baseline data were linked to data on hospital admissions and deaths; those with a history of whole/part prostatectomy $(\mathrm{n}=12309 ; 9.94 \%)$ or selected cancers $(\mathrm{n}=4742 ; 3.83 \%)$ were excluded (see online supplementary table S2). After exclusions, data on 106769 men were available for analyses.

The primary outcome was the first TURP performed for treating LUTS after recruitment into the study. Since our primary interest was non-cancer-related procedures, diagnosis of cancer, as defined in online supplementary table S3, was considered to be a competing risk and censored. If cancer diagnosis and TURP occurred in the same admission, the participant was censored for cancer, and the TURP was not counted as an outcome. Procedures $\operatorname{codes}^{12}$ identified as outcomes, in consultation with a urologist (author Patel), included: 37203-00 Transurethral resection of prostate, 37201-00 Transurethral needle ablation of prostate, 37203-02 Transurethral electrical vaporisation of prostate, 37207-00 Endoscopic laser ablation of prostate and 37207-01 Endoscopic laser excision of prostate. At least 1 of the above 5 procedures in any of the 50 procedure code fields in APDC was considered to be an outcome. Only nine individuals were found to have a suprapubic prostatectomy (code 37200-03) and two were found to have a retropubic prostatectomy (code 37200-04); these outcomes were considered to have insufficient numbers of events for reliable quantification of relationships and were therefore not included in the outcome measure.

In the analyses of incident procedures since baseline, eligible participants contributed person-years from the date of recruitment until admission date for TURP, 
cancer diagnosis date, date of death or end of follow-up (18 June 2014), whichever was the earliest. Age-standardised rates of incident TURP were calculated by age group $(45-54,55-64,65-74$ and $\geq 75)$ and by m-IPSS category $(0-2,3-5,6-8,9-11$ and 12-21). Incident TURP rates since baseline were age-standardised to the 2006 NSW population, in 5-year age groups, using the direct method. ${ }^{15}$

HRs for TURP in relation to baseline characteristics were estimated using Cox regression modelling. Three models were built: Model 1 adjusted for age (the underlying time variable); Model 2 additionally adjusted for region of birth, region of residence, smoking, alcohol consumption and BMI; Model 3 additionally adjusted for need as measured by m-IPSS. Sensitivity analysis looked at additional adjustment for annual household income.

Proportionality assumptions were verified using tests based on the Schoenfeld residuals (with a significance level of 0.00001 due to the large sample size); a stratified form of the model was used where covariates displayed non-proportionality of hazards. Missing values were included in the models as separate categories. Statistical tests were two-sided, using a significance level of 0.05 , unless otherwise stated. Analyses were carried out using SAS V.9.3 and stata v.12. Ethics approval for the study was obtained from the NSW Population and Health Services Research Ethics Committee and the Australian National University Human Research Ethics Committee.

\section{RESULTS}

Of the 106769 men included in the analyses, the majority $(63 \%)$ were aged <65 years; $3 \%$ reported severe LUTS symptoms (m-IPSS score 12-21), 18\% moderate symptoms (m=IPSS score $6-11$ ) and $80 \%$ no/mild symptoms (m-IPSS score 0-5); other characteristics of the sample are described in table 1.

There were 3416 incident procedures among 106769 men (median follow-up 5.8 years). Rates of TURP increased steadily with age, increasing from 1.78/1000 person-years among those aged 45-54 years to 11.40 / 100 person-years in those aged 75 or more (figure 1 ). Age-standardised rates of TURP increased with increasing severity of LUTS at baseline, increasing 14-fold from 2.15/1000 person-years with no/mild symptoms to $30.70 / 1000$ person-years with severe symptoms (figure 2). We found that $15 \%(429 / 2815)$ of those with severe symptoms at baseline went on to have a TURP over the median 5.8 years of follow-up, compared with $1 \%(816 / 74308)$ of those with no or mild symptoms.

In age-adjusted analyses, annual household income $\geq \$ 70000$ ( vs $<\$ 20000$ ), having private health insurance and living in major cities (vs more remote) were associated with a higher risk of TURP; current smoking (vs never smoking) was associated with a significantly lower risk of TURP; there were no significant differences according to educational attainment, region of birth or
Table 1 Characteristics of study participants at baseline

\begin{tabular}{|c|c|c|}
\hline & $\mathbf{n}$ & Per cent \\
\hline \multicolumn{3}{|l|}{ Age in years } \\
\hline $45-54$ & 31015 & 29 \\
\hline $55-64$ & 36462 & 34 \\
\hline $65-74$ & 23898 & 22 \\
\hline \multirow{2}{*}{\multicolumn{3}{|c|}{ m-IPSS* }} \\
\hline & & \\
\hline $0-2$ & 44790 & 48 \\
\hline $3-5$ & 29518 & 32 \\
\hline $6-8$ & 11760 & 13 \\
\hline $9-11$ & 4302 & 5 \\
\hline $12-21$ & 2815 & 3 \\
\hline \multicolumn{3}{|l|}{ Sociodemographic characteristics } \\
\hline Tertiary education & 27468 & 26 \\
\hline Born in Australia or New Zealand & 80308 & 76 \\
\hline Annual household income $\geq \$ 70000$ & 32123 & 36 \\
\hline $\begin{array}{l}\text { Private health insurance (Hospital/ } \\
\text { DVA) }\end{array}$ & 70152 & 66 \\
\hline Residing in major cities & 48885 & 46 \\
\hline \multicolumn{3}{|l|}{ Health behaviours } \\
\hline Never smoker & 51175 & 48 \\
\hline In highest tertile of physical activity & 35603 & 34 \\
\hline \multicolumn{3}{|l|}{ Health conditions } \\
\hline $\begin{array}{l}\text { Doctor-diagnosed cardiovascular } \\
\text { disease } \dagger\end{array}$ & 19943 & 19 \\
\hline Doctor-diagnosed heart disease & 15565 & 15 \\
\hline Doctor-diagnosed stroke & 3476 & 3 \\
\hline Doctor-diagnosed diabetes & 11075 & 10 \\
\hline $\begin{array}{l}\text { Doctor-diagnosed high blood } \\
\text { pressure }\end{array}$ & 38970 & 36 \\
\hline Had vasectomy & 27085 & 25 \\
\hline Severe erectile dysfunction & 13652 & 15 \\
\hline Requires help with daily tasks & 4796 & 5 \\
\hline Severe physical functioning limitation & 9828 & 10 \\
\hline $\begin{array}{l}\text { High psychological distress } \\
\text { (K10 score 22-50) }\end{array}$ & 6656 & 7 \\
\hline Poor self-rated health & 2263 & 2 \\
\hline Obese (BMI $30-50 \mathrm{~kg} / \mathrm{m}^{2}$ ) & 22077 & 22 \\
\hline
\end{tabular}

Percentages do not include missing cases in the denominators.

Number of missing cases: $\mathrm{m}$-IPSS $=13584$; education

attainment=1769; region of birth=897; annual household

income $=16733$; health insurance $=1$; region of residence $=88$;

cigarette smoking $=376$; sessions of physical activity $=3292$;

erectile dysfunction $=12705$; requires help with daily tasks $=4893$;

physical functional limitation $=11528$; psychological distress $=9452$;

self-rated health $=3218$; body mass index $=6684$; other variables $=0$

missing.

${ }^{*} \mathrm{~m}$-IPSS categories are based on its clinical ranges: $0-5$ (no/mild symptoms), 6-11 (moderate symptoms) and 12-21 (severe symptoms). The first two ranges are split into two equal subcategories, leaving the m-IPSS with the above five categories. †Participants were categorised as having cardiovascular disease (CVD) if they answered YES to any of the following 'Has your doctor ever told you that you have heart disease/stroke/blood clot (thrombosis)?'.

physical activity (figure 3). Adjusting additionally for sociodemographic and behavioural risk factors (Model 2) and need (LUTS severity, Model 3) resulted in little change in the overall pattern of results, except for annual household income $\geq \$ 70000$ (HR increased from 1.14 in Model 1 to 1.33 in Model 3). 
Figure 1 Rates of transurethral prostatectomy by age group. *Age standardised rate per 1000 person-years.
Figure 2 Rates of transurethral prostatectomy by modified International Prostate Symptom Score (m-IPSS) at baseline. ${ }^{*}$ Age standardised rate per 1000 person-years. ${ }^{* *}$ m-IPSS categories are based on its clinical ranges: $0-5$ (no/mild symptoms), 6-11 (moderate symptoms) and 12-21 (severe symptoms). The first two ranges were split into two equal subcategories, resulting in the above five m-IPSS categories. The rates corresponding to no/mild symptoms and moderate symptoms were 2.15 (2.01-2.30) respectively. and 10.06 (9.44-10.79),
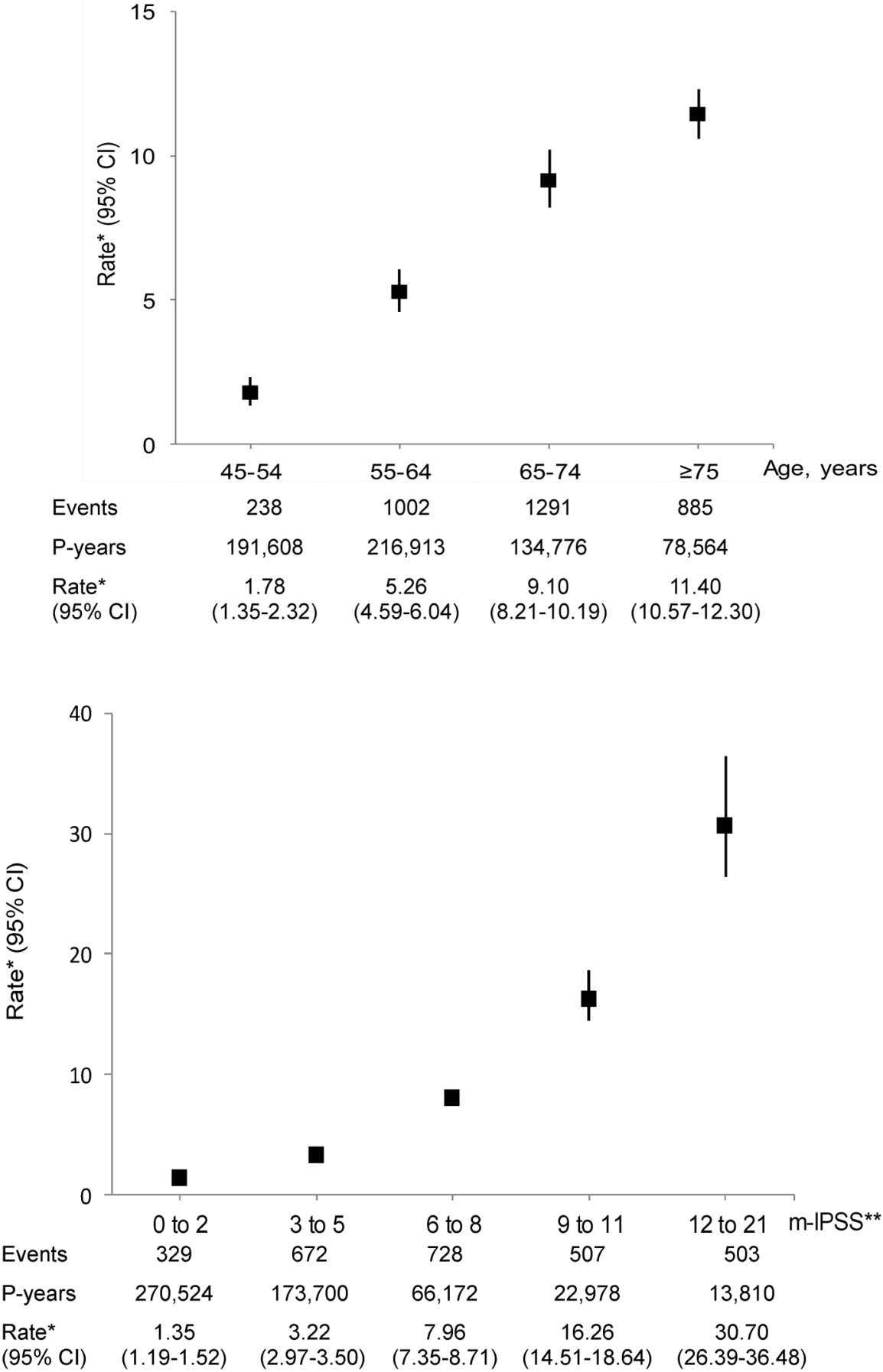

Regarding health conditions, there were no significant differences in TURP rates according to baseline BMI, diabetes, stroke, high blood pressure or cardiovascular disease (figure 4); adjusting additionally for sociodemographic and behavioural risk factors (Model 2) or need (Model 3) resulted in little change in the overall pattern of these results. In contrast, men reporting severe versus no physical functioning limitations, high versus low psychological distress or poor versus excellent self-rated health were $36-51 \%$ more likely to undergo procedures overall, but were $24-37 \%$ less likely to undergo procedures following additional adjustment for need (figure 4). Sensitivity analysis indicated that private health insurance remained a key factor relating to TURP even after adjustment for need and annual household income (see online supplementary table $\mathrm{S} 1$ ).

\section{DISCUSSION}

In this large, population-based cohort study, the rates of TURP varied up to 14-fold according to the severity of LUTS at baseline and increased steeply with increasing age. After accounting for age and the level of need, as measured by baseline LUTS, TURP rates were higher in men of higher incomes, living in major cities and with private health insurance, and were lower in those with 


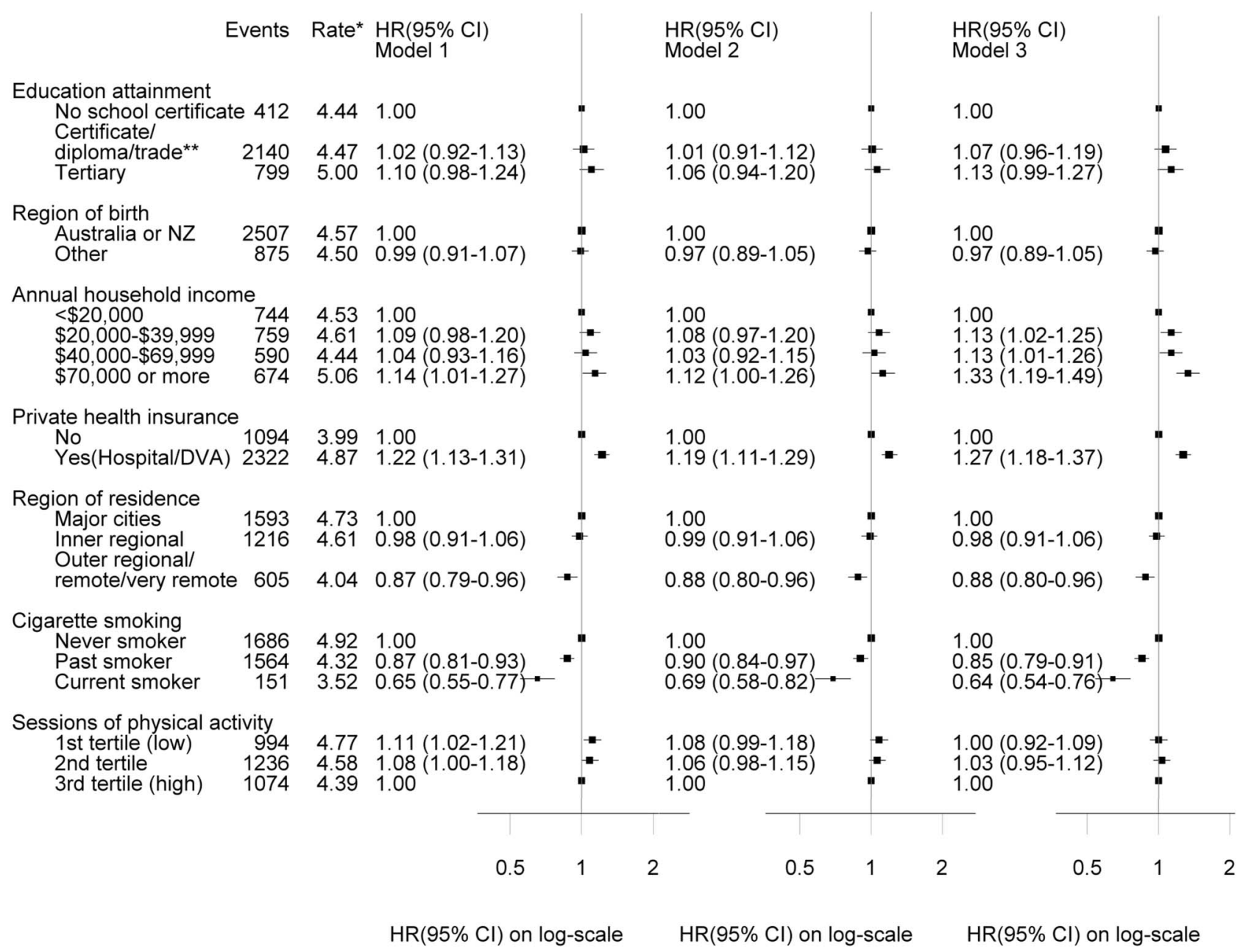

Figure 3 HRs for transurethral prostatectomy by demographic and behavioural characteristics.

*Age standardised rate per 1000 person-years. ${ }^{\star *}$ The subgroup 'certificate/diploma/trade' consists of the following four categories: school/intermediate certificate, higher school or leaving certificate, trade/apprenticeship and certificate/diploma. HRs by alcohol consumption are not presented due to violation of the proportionality assumption in the regression model. Model 1 is adjusted for age only. Model 2 is adjusted for age, alcohol consumption, smoking, BMI, region of birth and region of residence. Model 3 is adjusted for modified International Prostate Symptom Score in addition to the covariates in Model 2.

disabilities and with poorer overall health. However, the magnitude of the variation in surgery for LUTS observed according to baseline symptoms was far greater than that seen according to socioeconomic or other health-related factors.

The fact that the incidence and progression of LUTS, including surgical intervention, increase with age is well established. ${ }^{6}{ }^{16-19}$ While it seems obvious that greater LUTS at baseline would be a key predictor of subsequent surgical intervention, quantitative data on this are surprisingly limited, particularly when the population burden and costs of LUTS are considered. We identified two studies presenting quantitative data on the relationship of baseline symptoms to relevant outcomes, both from the USA. Data from 16219 men from a Health Maintenance Organisation with baseline health data collected in 1971-1972 demonstrated a strong relationship of five urological symptoms to surgical treatment for benign prostatic hypertrophy, with relative risks of 4.6-
5.8 for severe versus no symptoms. ${ }^{6}$ Evidence from 5986 men participating in the placebo arm of the Prostate Cancer Prevention Trial showed a 10-fold difference between those with mild versus severe baseline LUTS in the composite outcome of surgical or medical treatment for benign prostatic hypertrophy or sustained LUTS. ${ }^{5}$

The data presented here provide contemporary estimates that are based on a sample that is an order of magnitude larger than available previously and relates to the general population, rather than to a trial or health maintenance organisation setting. It is also the first evidence, to the best of our knowledge, from a country with universal healthcare. The large numbers permit estimates of incidence based on fine gradations of baseline LUTS, with narrow CIs. The combination of detailed data on LUTS at baseline and individual data on a wide range of socioeconomic, health behaviour and health status factors allowed more detailed consideration of the targeting of TURP, including consideration 


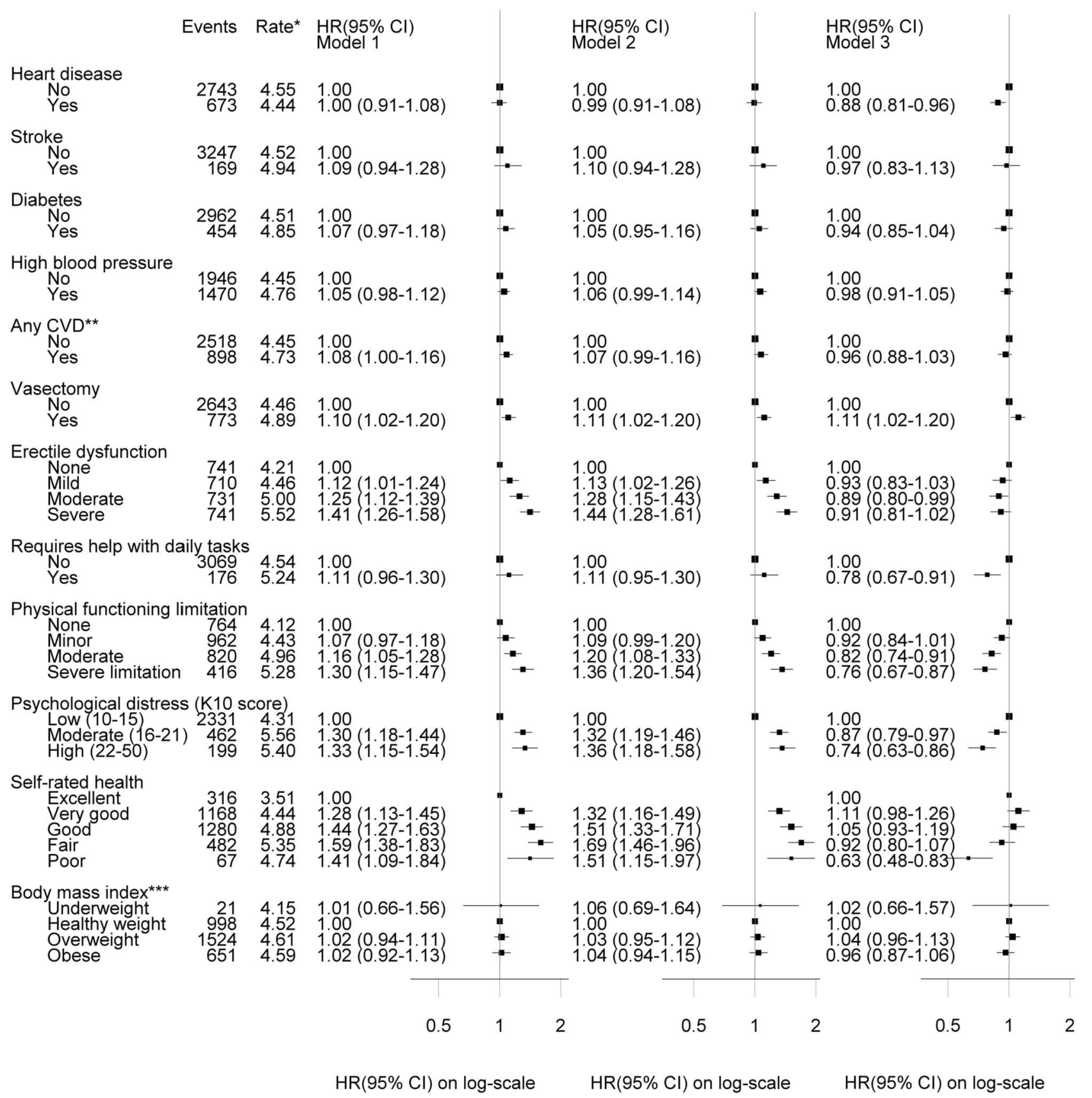

Figure 4 HR for transurethral prostatectomy by health conditions. *Age standardised rate per 1000 person-years. ${ }^{*}$ Participants were categorised as having cardiovascular disease (CVD) if they answered YES to any of the following questions: 'Has your doctor ever told you that you have: heart disease/stroke/blood clot (thrombosis)?'. ${ }^{* *}$ Body mass index categories are Underweight $\left(15-<18.5 \mathrm{~kg} / \mathrm{m}^{2}\right)$, Healthy weight $\left(18.5-<25 \mathrm{~kg} / \mathrm{m}^{2}\right)$, Overweight $\left(25-<30 \mathrm{~kg} / \mathrm{m}^{2}\right)$ and Obese $\left(30-50 \mathrm{~kg} / \mathrm{m}^{2}\right)$. Model 1 is adjusted for age only. Model 2 is adjusted for age, alcohol consumption, smoking, BMI, region of birth and region of residence. Model 3 is adjusted for modified International Prostate Symptom Score in addition to the covariates in Model 2.

of any variation that persists after accounting for healthcare need, as measured by LUTS at baseline.

The study demonstrates prospectively that the key drivers for TURP are baseline symptoms and age, indicating that surgical interventions are being targeted at those with the greatest need. At the same time, the $\sim 30 \%$ higher TURP rates observed in those with higher income and private health insurance, after accounting for need, suggest some inequity in the use of the procedure. Previous research shows that severe LUTS is more common in men of low socioeconomic status. ${ }^{7} 20$ In contrast to evidence on risk factors for symptoms, TURP is associated with higher socioeconomic status; risk factor associations of socioeconomic factors with TURP have not, to the best of our knowledge, been shown before. In previous work, both current and past smokers had higher odds of severe LUTS than never smokers. $^{7}$ The lower risk of TURP observed in this study 
among current smokers is not explained by differences in LUTS severity according to smoking status; the reason for this is unclear but may relate to lower socioeconomic status and reduced health-seeking behaviour. Current findings are consistent with postulated lower rates of TURPs in rural Australia. ${ }^{21}$

The findings also demonstrate, for the first time according to the best of our knowledge, that while those with poorer health are more likely to receive TURPs overall, they are less likely to receive such interventions once their level of need is taken into account. This finding that ill health and disability are related to LUTS is consistent with previous data. ${ }^{70}$ The reason for the lower levels of intervention in those with LUTS and ill health or disability is unclear; contributing factors may include frailty and risk of undergoing an operation, lack of prioritisation of LUTS in the face of other health problems and persisting inequity in the use of the procedure.

Since BPE is a common condition among older men, with an estimated prevalence of $6 \%$ in men aged $45-$ 64 years rising to $24 \%$ in men aged $\geq 75$ years, ${ }^{22}$ LUTS are commonly perceived as part of natural ageing. Despite the high prevalence of men who experience symptoms, our findings are consistent with the published evidence that only a small proportion of those with severe symptoms receive medical intervention. ${ }^{23}$ There are four types of treatment options available: watchful waiting (education, reassurance, periodic review and lifestyle advice), pharmacotherapy (using $\alpha$-blockers or 5 - $\alpha$-reductase inhibitors), minimally invasive surgical therapies (such as TUNA/TUVP) and surgery (TURP or laser ablation/excision of prostate). ${ }^{24}$ Treatment options are chosen depending on disease severity, impact on quality of life, patient preference, presence of complications and fitness for surgery. ${ }^{25}$ TURP is considered to be the 'gold standard' of care and is by far the most common procedure performed for treating non-cancer-related LUTS, particularly hyperplasia of the prostate. ${ }^{26}$ However, increasing numbers of patients are being treated with laser surgery over time. ${ }^{27}$

All five procedures investigated were subsidised by the Australian government throughout the study period. However, nearly two-thirds of all prostatectomy procedures are performed in private hospitals, ${ }^{28}$ possibly due to the long waiting time for elective surgery for prostatectomy in public hospitals. The median waiting time in 2011-2012 was 42 days, ranging from 56 days for outer regional areas to 41 days for major cities, and from 37 days for people living in areas classified as the least socioeconomically disadvantaged to 49 days for the most disadvantaged. ${ }^{28}$ These inequalities in access to care are likely to at least partly explain the higher need-adjusted rates among people in higher income households and who hold private health insurance found in this study.

The large sample size of this study enabled us to look at factors related to TURP comprehensively, adjusting for a range of potential confounding factors. The ability to link to administrative records was a strength of the study, allowing virtually complete ascertainment of outcomes over time and enhanced identification of people with previous cancer (so that they could be excluded from the study) and those developing bowel/genital/urinary-tract cancer during follow-up (censored as a competing risk). Subgroup analyses enabled us to investigate whether TURP varied significantly between the different subgroups of sociodemographic and health factors.

Limitations of the study include non-availability of data on the management of symptoms in clinical practice, lack of information on contraindications to surgical intervention and the use of self-reported survey data. Ascertainment of LUTS was based on self-report using a measure calibrated against the validated and widely used IPSS. The range of other factors was based on self-reported data, which have inherent limitations, but were nonetheless the most practicable and reliable available. It should be noted that although the 45 and Up Study is, like the vast majority of cohort studies, not strictly representative of the general population, the results presented here are based on internal comparisons within the cohort and are likely to be reliable. ${ }^{29}$ Health need was measured by LUTS at baseline and it is likely that some individuals experienced progression of LUTS during the 5.8-year mean follow-up period. This means that the incidence of procedures associated with lower severity scores may be overestimated and the estimates in Model 3 underadjusted for need. This study investigated the factors related to TURP. No information was available on whether the men were referred to a urologist, whether the procedures were offered or surgery was refused. Some people with LUTS may be managed using catheterisation, including: those considered unsuitable for surgical intervention due to their health condition, those refusing surgical intervention and those with LUTS due to causes other than bladder outlet obstruction. Furthermore, catheterisation is included in the management of many conditions other than LUTS; this study focused on surgical interventions specific to LUTS, including TURP. Information about barriers to access services, such as those due to geographic location of services and out-of-pocket expenses, was not available.

The findings of this study have a range of implications. They are reassuring in that they indicate a degree of alignment between symptoms and treatment designed to treat those symptoms. At the same time, they suggest that there are likely to be unmet health needs relating to LUTS in men experiencing greater disadvantage, ill health and disability. Greater consideration of the needs of men in this situation by policymakers and practitioners is warranted. Data on the additional factors likely to explain the observed levels of inequality would be useful. As it stands, the observed greater use of TURP, after accounting for need, in more socioeconomically advantaged groups could be due to overuse in this group, underuse in less advantaged groups or a mixture 
of the two. Future work quantifying the likely unmet need for LUTS treatment and providing insights into how to ensure appropriate access to such treatment, particularly for those with physical health problems and issues with accessing care, would be of value.

In conclusion, TURP is substantively targeted at men with the highest levels of symptoms. However, the lower use of TURP, relative to need, among men who are disadvantaged and likely to have less access to surgery suggests inequality. The lower use of TURP in men experiencing poorer health and with disabilities is likely to be the result of a combination of factors, including frailty, risk of surgery and inequity.

Acknowledgements This research was completed using data collected through the 45 and Up Study (http://www.saxinstitute.org.au). The 45 and Up Study is managed by the Sax Institute in collaboration with major partner Cancer Council NSW; and partners: the National Heart Foundation of Australia (NSW Division); NSW Ministry of Health; NSW Government Family \& Community Services-Carers, Ageing and Disability Inclusion; and the Australian Red Cross Blood Service. The authors thank the many thousands of people participating in the 45 and Up Study.

Contributors EB and GJ conceived the project. GJ wrote the analysis plan. GJ and KS conducted the analysis and drafted the initial version of the manuscript. All authors contributed to a review of the analysis plan, interpretation of results and revisions of the manuscript.

Funding This study is funded by the Australian National Health and Medical Research Council (NHMRC project grant number 1024450) and by the NHMRC Centre for Research Excellence in Medicines and Ageing. EB is supported by the NHMRC.

\section{Competing interests None declared.}

Ethics approval NSW Population and Health Services Research Ethics Committee and the Australian National University Human Research Ethics Committee.

\section{Provenance and peer review Not commissioned; externally peer reviewed.}

Data sharing statement Information about data access and governance policies is available at: https://www.saxinstitute.org.au/our-work/45-up-study/ for-researchers/

Open Access This is an Open Access article distributed in accordance with the Creative Commons Attribution Non Commercial (CC BY-NC 4.0) license, which permits others to distribute, remix, adapt, build upon this work noncommercially, and license their derivative works on different terms, provided the original work is properly cited and the use is non-commercial. See: http:// creativecommons.org/licenses/by-nc/4.0/

\section{REFERENCES}

1. Parsons JK. Benign prostatic hyperplasia and male lower urinary tract symptoms: epidemiology and risk factors. Curr Bladder Dysfunct Rep 2010;5:212-18.

2. AlHW \& Australasian Association of Cancer Registries. Cancer in Australia: an overview. 2006. Cancer series no. 37. AlHW Cat. no. CAN 32. Canberra: AlHW, 2007.

3. Kirby RS, Kirby M, Fitzpatrick JM. Benign prostatic hyperplasia: counting the cost of its management. BJU Int 2010;105:901-2.

4. Wei JT, Calhoun E, Jacobsen SJ. Urologic diseases in America project: benign prostatic hyperplasia. J Urol 2005;173:1256-61.

5. Schenk JM, Hunter-Merrill R, Zheng Y, et al. Should modest elevations in prostate-specific antigen, International Prostate Symptom Score, or their rates of increase over time be used as surrogate measures of incident benign prostatic hyperplasia? Am J Epidemiol 2013:178:741-51.
6. Sidney S, Quesenberry C Jr, Sadler MC, et al. Risk factors for surgically treated benign prostatic hyperplasia in a prepaid health care plan. Urology 1991;38:13-19.

7. Smith DP, Weber MF, Soga K, et al. Relationship between lifestyle and health factors and severe lower urinary tract symptoms (LUTS) in 106,435 middle-aged and older Australian men: population-based study. PLOS ONE 2014;9:e109278.

8. Latz I, Weber M, Korda R, et al. Lower urinary tract symptoms in relation to region of birth in 95,393 men living in Australia: the 45 and Up Study. World J Urol 2013;31:673-82.

9. Fritschi L, Tabrizi J, Leavy J, et al. Risk factors for surgically treated benign prostatic hyperplasia in Western Australia. Public Health 2007;121:781-9.

10. Banks E, Redman S, Jorm L, et al. Cohort profile: the 45 and up study. Int J Epidemiol 2008;37:941-7.

11. National Centre for Classification in Health. International statistical classification of diseases and related health problems, 10th Revision, Australian Modification (ICD-10-AM). 5th edn. Sydney: National Centre for Classification in Health, 2006.

12. National Casemix and Classification Centre (NCCC). The Australian classification of health interventions (ACHI). 8th edn. Tabular list of interventions and Alphabetic index of interventions. Wollongong: NCCC, Australian Health Services Research Institute, University of Wollongong, 2012.

13. Hays RD, Liu H, Spritzer K, et al. Item response theory analyses of physical functioning items in the medical outcomes study. Med Care 2007;45(Suppl 1):S32-8.

14. ABS. Information paper: use of the Kessler Psychological distress scale in ABS health surveys, Cat. No. 4817.0.55.001. Canberra: Australian Bureau of Statistics, 2001.

15. Fay MP, Feuer EJ. Confidence intervals for directly standardized rates: a method based on the gamma distribution. Stat Med 1997;16:791-801.

16. Giovannucci E, Rimm EB, Chute CG, et al. Obesity and benign prostatic hyperplasia. Am J Epidemiol 1994;140:989-1002.

17. Verhamme KM, Dieleman JP, Bleumink GS, et al. Incidence and prevalence of lower urinary tract symptoms suggestive of benign prostatic hyperplasia in primary care-the Triumph project. Eur Urol 2002;42:323-8.

18. Platz EA, Joshu CE, Mondul AM, et al. Incidence and progression of lower urinary tract symptoms in a large prospective cohort of United States men. J Urol 2012;188:496-501.

19. Irwin DE, Milsom I, Kopp Z, et al. Prevalence, severity, and symptom bother of lower urinary tract symptoms among men in the EPIC study: impact of overactive bladder. Eur Urol 2009;56:14-20.

20. Coyne KS, Sexton CC, Thompson CL, et al. The prevalence of lower urinary tract symptoms (LUTS) in the USA, the UK and Sweden: results from the Epidemiology of LUTS (EpiLUTS) study. BJU Int 2009;104:352-60.

21. $\mathrm{Ng} \mathrm{JQ}$, Hall SE, Holman CDAJ, et al. Inequalities in rural health care: differences in surgical intervention between metropolitan and rural Western Australia. ANZ J Surg 2005;75:265-9.

22. Charles J, Valenti L, Britt $\mathrm{H}$. BPH-management in general practice. Aust Fam Physician 2011;40:757-7.

23. Pearce I. NICE Quality Standard: full assessment is key for men with lower urinary tract symptoms. 2014. http://www. guidelinesinpractice.co.uk/feb_14_pearce_ogu_feb14\#.VjbB-EajcvU

24. Woo HH, Gillman MP, Gardiner R, et al. A practical approach to the management of lower urinary tract symptoms among men. Med $J$ Aust 2011;195:34-9.

25. Beduschi MC, Beduschi R, Oesterling JE. Alpha-blockade therapy for benign prostatic hyperplasia: from a nonselective to a more selective alpha1A-adrenergic antagonist. Urology 1998;51:861-72.

26. Thangasamy IA, Chalasani V, Bachmann A, et al. Photoselective vaporisation of the prostate using $80-\mathrm{W}$ and $120-\mathrm{W}$ laser versus transurethral resection of the prostate for benign prostatic hyperplasia: a systematic review with meta-analysis from 2002 to 2012. Eur Urol 2012;62:315-23.

27. Stafinski T, Menon D, Harris K, et al. Photoselective vaporization of the prostate for the treatment of benign prostatic hyperplasia. Can Urol Assoc J 2008;2:124-34.

28. AlHW. Australian hospital statistics 2011-12. Health services series 50. Cat. no. HSE 134. Canberra: AlHW, 2013.

29. Mealing NM, Banks E, Jorm LR, et al. Investigation of relative risk estimates from studies of the same population with contrasting response rates and designs. BMC Med Res Methodol 2010;10:26. 